\title{
Meninos com queixa escolar: autopercepções, desempenho e comportamento
}

\author{
Boys with complaints about school: self-perception, \\ achievement, and behavior
}

\author{
Andresa Aparecida FERREIRA \\ Karina de Melo CONTE² \\ Edna Maria MARTURANO
}

\begin{abstract}
Resumo
Este estudo objetivou caracterizar as autopercepções de meninos com queixa escolar, bem como verificar associações entre essa percepção e indicadores de desempenho escolar e problemas de comportamento. Em uma clínica-escola vinculada ao Sistema Único de Saúde, foram avaliados 50 meninos com queixa escolar, na faixa etária de 6 a 11 anos. Foram utilizados os seguintes instrumentos: Teste de Matrizes Progressivas de Raven, Teste de Desempenho Escolar, Roteiro de Avaliação do Senso de AutoEficácia, Escala Infantil Piers-Harris de Autoconceito, Inventário de Comportamentos da Infância e Adolescência. Nos resultados, o autoconceito global e o senso de autoeficácia mostraram correlação modesta, mas significativa, com a medida global de desempenho. O autoconceito na categoria felicidade correlacionou-se com todos os indicadores de desempenho, ao passo que o autoconceito na categoria status intelectual e acadêmico não se associou com desempenho. As autopercepções não mostraram associação com problemas de comportamento. Novas pesquisas são necessárias para esclarecer esse padrão de associações.
\end{abstract}

Unitermos: Autoconceito. Autoeficácia. Comportamento. Desempenho Escolar.

\begin{abstract}
The aim of this study was to characterize the self-perceptions of boys experiencing difficulties with school, as well as to ascertain the connection between these perceptions and indices of academic performance and behavioral problems. In a university-based clinic linked to the public health system, we assessed 50 boys, with complaints about school, between the ages of 6 and 11 . The instruments used were, Raven Colored Progressive Matrices Test, School Performance Test, Self-Efficacy Assessment Form, Piers-Harris Children's Self-Concept Scale, Child Behavior Checklist. Results showed both global self-concept and self-efficacy to correlate modestly but significantly with academic performance measures. The self-concept of happiness correlated with all measures of school performance, while self-concept in terms of intellectual and academic status did not correlate with school performance at all. Self-perceptions bore no association with behavioral problems. There is a need for further studies to clarify this pattern of associations.
\end{abstract}

Uniterms: Selfconcept. Selfefficacy. Behavior. Academic achievement.

\section{$\boldsymbol{\nabla \nabla \nabla \nabla}$}

1 Universidade Estadual de Campinas, Faculdade de Educação. Campinas, SP, Brasil.

2 Universidade de São Paulo, Faculdade de Filosofia, Ciências e Letras. Batatais, SP, Brasil.

3 Universidade de São Paulo, Faculdade de Medicina de Ribeirão Preto, Departamento de Neurociências e Ciências do Comportamento. R. Tenente Catão Roxo, 14049-900 Ribeirão Preto, SP, Brasil. Correspondência para/Correspondence to: E.M. MARTURANO. E-mail: <emmartur@fmrp.usp.br>. 
No processo de aprendizagem, a maior eficiência para a aquisição de informação depende não somente do conjunto de habilidades e conhecimentos prévios do indivíduo, mas também do conhecimento e controle que possui sobre seus processos cognitivos, ou seja, de suas habilidades metacognitivas (Martin \& Marchesi, 1995). O funcionamento da inteligência e da motivação exige a utilização desses processos cognitivos e é sensível a fatores sociais ou emocionais. Com isso, pode-se considerar que a capacidade intelectual fixa certos limites à capacidade de aprendizagem do aluno em cada momento, ao passo que a motivação é responsável pela utilização mais ou menos completa desse potencial, ou pela orientação dos investimentos intelectuais em tarefas ou domínios diferentes (Fontaine, 1995).

Nesse sentido, pode-se supor que a criança terá maior motivação para enfrentar a situação de aprendizagem escolar na medida em que tiver uma percepção positiva de seu potencial intelectual e de sua capacidade para aprender os conteúdos escolares e obter sucesso. Em contrapartida, a motivação para aprender será menor na medida em que a criança não se perceber com os recursos necessários para tal aprendizagem. Dentre as variáveis afetivas, relacionadas às autopercepções que podem ter relação com o desempenho acadêmico, destacam-se o autoconceito e a autoeficácia.

O autoconceito pode ser definido como um conjunto de atribuições cognitivas que um indivíduo faz a respeito de si, de suas características pessoais e de seu comportamento em situações objetivas (Candieux, 1996). É um construto multidimensional, no sentido de que envolve atribuições em domínios específicos, como comportamento, aparência, desempenho acadêmico e aceitação social. Seu desenvolvimento é influenciado pela opinião de pessoas importantes, como os pais, professores e colegas (Gest, Rulison, Davidson \& Welsh, 2008), e ele se torna mais diferenciado e estável à medida que a criança cresce (Donohue, Wise, Romski, Henrich \& Sevcik, 2010).

O senso de autoeficácia é o julgamento que o indivíduo faz acerca de sua capacidade de mobilizar recursos cognitivos e ações de controle sobre eventos e demandas do meio, para atingir certo grau de performance (Bandura, 2006). No contexto escolar, a autoeficácia acadêmica diz respeito aos julgamentos 444 que o aluno faz sobre sua própria capacidade de orga- nizar e executar cursos de ação, para atingir determinados tipos de desempenho educacional (Carrol et al., 2009). Apontando semelhanças e diferenças entre os autoconceito e autoeficácia, Bong e Skaalvik (2003) assinalam que o senso de autoeficácia é mais dependente do contexto específico em que a pessoa faz o autojulgamento e, por essa razão, espera-se que seja menos estável e mais maleável que o autoconceito.

Pesquisas no contexto escolar sobre a relação entre autopercepções e desempenho acadêmico mostram uma associação positiva entre autoconceito e diferentes habilidades acadêmicas, em amostras de vários níveis de idade, escolaridade e status socioeconômico (Valentine, Dubois \& Cooper, 2004). A relação parece ser recíproca ao longo do tempo, e mais acentuada quando o autoconceito e o desempenho são avaliados no mesmo domínio - línguas ou matemática, por exemplo (Marsh \& Craven, 2006). Quanto à autoeficácia, pesquisas com adolescentes a partir de 11 anos mostram que um positivo senso de autoeficácia acadêmica prediz aprendizado e desempenho escolar (Carrol et al., 2009; Fast et al., 2010).

A literatura científica brasileira oferece evidências de associação entre autopercepções e desempenho escolar em alunos do ensino fundamental (Carneiro, Martinelli \& Sisto, 2003; Cia \& Barham, 2009; Cunha, Sisto \& Machado, 2006, 2007; Jacob, 2001; Moraes \& Cunha, 2007; Silva \& Alencar, 1984; Souza \& Brito, 2008). Os resultados nem sempre são comparáveis, devido à adoção de diferentes conjuntos de domínios e instrumentos.

Utilizando medida global de autoconceito, Silva e Alencar (1984) encontraram, em alunos da 4a série, correlação significativa entre autoconceito e rendimento acadêmico. Da mesma forma, no estudo de Cia e Barham (2009) com alunos de $1^{\text {a }}$ e $2^{\text {a }}$ série, um escore global de autoconceito do Self-Description Questionnaire (SDQ) correlacionou-se positivamente com a avaliação de desempenho feita pelo professor e também com escores em leitura, escrita e aritmética do Teste de Desempenho Escolar (TDE). As autoras obtiveram ainda correlação negativa entre o autoconceito global e comportamentos problemáticos avaliados por pais e professores.

Outros estudos adotaram uma perspectiva multidimensional de autoconceito. Jacob (2001) usou a Escala Infantil Piers-Harris, que avalia seis categorias de 
autoconceito, para comparar dois grupos de crianças diferenciadas quanto ao desempenho escolar. O grupo com bom desempenho mostrou melhor autoconceito nas categorias status intelectual e acadêmico e comportamento, além de melhor senso de autoeficácia acadêmica e melhor comportamento, este avaliado pelas mães.

Carneiro et al. (2003), em um delineamento de comparação de grupos, investigaram a relação entre a dificuldade na escrita de palavras e o autoconceito nos domínios pessoal, social, familiar e escolar de crianças da 3a série. Encontraram que a dificuldade na escrita está inversamente relacionada com o autoconceito escolar. Já Cunha et al. (2006; 2007), em estudo correlacional com alunos de $2^{a}, 3^{a}$ e $4^{a}$ série segregados por sexo, usando a Escala de Autoconceito Infanto-Juvenil, não reproduziram essa associação, mas obtiveram correlação negativa entre dificuldade na escrita de palavras e autoconceito social (meninos da $2^{\mathrm{a}}$ e da $4^{\mathrm{a}}$ série) e familiar (meninas da 2a série). Também obtiveram correlações positivas entre reconhecimento de palavras e autoconceito pessoal e social, com variações por sexo e série. O autoconceito escolar teve correlação com o reconhecimento de palavras apenas para as meninas da $2^{a}$ série. Por outro lado, Moraes e Cunha (2007) encontraram correlações positivas entre os resultados do instrumento de reconhecimento de palavras e todos os domínios da Escala de Autoconceito Infanto-Juvenil, em crianças da mesma população escolar.

Em resumo, os estudos brasileiros confirmam genericamente a associação entre autopercepções e desempenho escolar, porém não são homogêneos quanto ao domínio do autoconceito que se associa ao desempenho. Assim, a correspondência entre desempenho e autoconceito acadêmico é evidenciada por Jacob (2001) e Carneiro et al. (2003), mas não por Cunha et al. (2006), ao passo que na pesquisa de Moraes e Cunha (2007) tanto o autoconceito escolar como o pessoal, o social e o familiar correlacionam-se com um indicador específico de desempenho. Deve-se ressaltar, nos estudos baseados em correlações, que estas, embora significativas, são baixas, em geral não superiores a 0,30.

Crianças encaminhadas para programas de apoio psicopedagógico, dentro ou fora da escola, em razão do baixo desempenho escolar, podem estar particularmente vulneráveis a desenvolver autopercepções negativas. Além de terem maior visibilidade como alunos "fracos" em razão do encaminhamento, elas frequentemente são vistas como alunos que têm problemas de comportamento associados à dificuldade no aprendizado escolar (Marturano, Toller \& Elias, 2005). Essas opiniões desfavoráveis quanto a seu desempenho e comportamento podem ser internalizadas pela criança, dado que tanto a experiência direta como a opinião expressa por pais, professores e colegas têm um papel decisivo no desenvolvimento das autopercepções (Gest, Rulison, Davidson \& Welsh, 2008).

Este artigo trata do autoconceito e do senso de autoeficácia em crianças encaminhadas para serviço de apoio psicopedagógico, em associação com desempenho acadêmico e comportamento. A expressão "queixa escolar" é aqui usada para designar a condição particular em que essas crianças se encontram, de terem sido identificadas como alguém que precisa de acompanhamento fora da sala de aula, por apresentar dificuldade no aprendizado escolar.

Algumas pesquisas sobre autopercepções em crianças de 7 a 12 anos, identificadas com a queixa escolar, foram realizadas no Brasil, empregando a Escala Infantil Piers-Harris de Autoconceito. Stevanato, Loureiro, Linhares e Marturano (2003), comparando crianças em seguimento psicopedagógico clínico com crianças sem queixa escolar, verificaram que as primeiras tinham autoconceito significativamente mais negativo, não só no escore global e na categoria status intelectual e acadêmico, mas também nas categorias comportamento, ansiedade, popularidade e felicidade.

Okano, Loureiro, Linhares e Maturano (2004) encontraram resultados semelhantes, ao comparar crianças com desempenho pobre, que frequentavam além do ensino regular um programa complementar denominado Ensino Alternativo, e crianças sem dificuldade escolar, que frequentavam apenas o ensino regular. As crianças sem dificuldades obtiveram escores de autoconceito nas subescalas status intelectual e acadêmico, comportamento, ansiedade e popularidade significativamente mais altos do que os colegas que recebiam apoio no Ensino Alternativo.

Na mesma direção, Medeiros (2004) verificou que crianças com queixa escolar, atendidas em uma clínica de psicologia, quando comparadas a um grupo com 
bom desempenho acadêmico, apresentaram autoconceito global e senso de autoeficácia mais negativos. Elas mostraram autoconceito mais negativo também nos domínios acadêmico, comportamental, de ansiedade e popularidade, confirmando achados prévios de Medeiros, Loureiro, Linhares e Marturano (2000). De acordo com a percepção das mães, essas crianças tinham mais problemas de comportamento.

As pesquisas citadas sugerem certa vulnerabilidade das crianças identificadas com a queixa escolar, no sentido de desenvolverem autopercepções negativas. Elas não apenas têm autopercepções desfavoráveis no domínio escolar, como outros estudantes com fraco desempenho. Além disso, as crianças atendidas em serviços de apoio psicopedagógico parecem prejudicadas em outros domínios do autoconceito, relacionados ao comportamento, às emoções e às relações interpessoais.

Levando em conta os achados dessas pesquisas, o presente estudo focaliza, em um contexto clínico, as autopercepções de meninos com queixa escolar. O foco no gênero masculino se deve ao predomínio de meninos na população clínica com queixa escolar (American Psychiatric Association - APA, 1994; Marturano et al., 2005), bem como a indícios de apresentarem eles autoconceito mais prejudicado (Silva \& Fleith, 2005).

O objetivo do trabalho foi caracterizar, quanto ao autoconceito e à autoeficácia, meninos com queixa de dificuldade de aprendizagem, bem como verificar associações entre essas autopercepções e indicadores de desempenho escolar e problemas de comportamento. O principal interesse na realização da pesquisa era verificar, nesse segmento específico: (a) se seriam reproduzidas as associações, encontradas na população escolar, entre desempenho, autoconceito acadêmico e autoeficácia acadêmica; (b) se seriam encontradas correlações entre desempenho e autoconceito em domínios relacionados ao comportamento, às emoções e às relações interpessoais, conforme sugerido pelas pesquisas prévias que compararam crianças com e sem queixa escolar.

\section{Método}

O estudo foi realizado em uma clínica de psico- crianças encaminhadas por profissionais do Sistema Único de Saúde (SUS), com queixa de dificuldades de aprendizagem. A clínica está vinculada a um hospital universitário, em cidade do interior paulista com aproximadamente 500 mil habitantes. O projeto foi aprovado pelo Comitê de Ética em Pesquisa da Faculdade de Filosofia, Ciências e Letras da Universidade de São Paulo - campus de Ribeirão Preto, sob protocolo no 110/2003, em 30/03/2004, e todos os responsáveis pelos participantes assinaram um Termo de Consentimento Livre e Esclarecido, antes de sua inclusão na amostra.

Foram avaliadas 50 crianças do sexo masculino, na faixa etária de 6 anos a 10 anos e 11 meses, que cursavam da 1a à 4a série em escolas da rede pública e particular dos municípios de Ribeirão Preto e região.

Os instrumentos utilizados na avaliação foram: a) Matrizes Progressivas Coloridas de Raven, escala especial que avalia o nível intelectual das crianças, tomando-se as normas brasileiras de Angelini, Alves, Custódio e Duarte (1999); b) Teste de Desempenho Escolar -TDE (Stein, 1994), que avalia o nível de desempenho escolar em três escalas - escrita, aritmética e leitura -, fornecendo a classificação da criança em relação à série escolar; c) Escala Infantil Piers-Harris de Autoconceito 'O que eu sinto sobre mim mesmo' (Jacob, 2001), formada por 80 itens, cada um dos quais recebe escore "um" quando a resposta da criança indica autoconceito positivo. O escore total é obtido por meio da soma de todos os itens. A escala ainda fornece índices de autoconceito em seis categorias: comportamento, status intelectual e acadêmico, aparência física e atributos, ansiedade, popularidade, felicidade e satisfação; d) Roteiro de Avaliação de Auto-Eficácia, procedimento desenvolvido por Loureiro e Medeiros (2004), que focaliza a percepção da criança quanto a seu desempenho acadêmico e capacidade de realização. É composto por 20 afirmativas, que a criança avalia serem verdadeiras ou falsas em relação a seu desempenho escolar. Para cada resposta favorável a um senso de autoeficácia positivo, é atribuído um ponto, sendo o escore total obtido pela somatória de pontos; f) Inventário de Comportamentos da Infância e Adolescência - (CBCL) (Achenbach, 1991), que avalia problemas de comportamento em crianças de 4 a 18 anos, a partir de informações fornecidas pelos pais. Os escores brutos são convertidos em escores T, que permitem classificar os problemas de uma criança 
em três níveis - normal, limítrofe e clínico -, de acordo com normas americanas. No presente estudo, foi utilizada a versão adaptada no Brasil por Bordin, Mari e Caeiro (1995).

A coleta dos dados foi inserida na rotina da clínica. As entrevistas com a criança e suas mães/responsáveis foram feitas por uma pedagoga com especialização em psicopedagogia, auxiliada por uma psicóloga para a aplicação e cotação do Teste de Raven. Todos os instrumentos foram aplicados individualmente. O CBCL foi preenchido durante a primeira consulta com a mãe/ responsável. Os demais instrumentos foram aplicados em duas sessões individuais com a criança, uma para aplicação do Raven, e outra para aplicação do TDE, do Roteiro de Auto-Eficácia e da Escala de Autoconceito, nessa ordem.

Na análise quantitativa, foram calculadas estatísticas descritivas (porcentagem, medianas), bem como correlações (rho de Spearman) entre os escores dos diferentes instrumentos utilizados. As correlações foram calculadas sobre os escores brutos, exceto para o Raven, com resultados expressos em percentis.

\section{Resultados}

Na avaliação feita pelo Raven, 72\% das crianças tiveram pontuação em torno da média ou superior a ela (percentil 25 a 90), enquanto 28\% delas se classificaram no percentil 10 ou abaixo dele. No TDE, o maior percentual de crianças se situou no nível inferior: 90\% no subteste de escrita, 78\% em leitura, 76\% em aritmética e $80 \%$ no escore total do teste. Quanto ao comporta- mento avaliado pelo CBCL, 48\% das crianças apresentaram problemas de comportamento em nível clínico, e, de acordo com a percepção das mães, 70\% se encontravam em situação de risco (limítrofe ou clínico).

A Tabela 1 apresenta as medianas dos escores obtidos pelos participantes nas escalas de autoconceito e autoeficácia. Para comparação, foram incluídas na Tabela as medianas de grupos de crianças sem dificuldade escolar, encontradas por Jacob (2001), Okano (2001) e Medeiros (2004) que usaram os mesmos instrumentos utilizados nesta pesquisa para avaliar autoconceito e autoeficácia.

Observa-se (Tabela 1) que as medianas encontradas neste estudo tendem a ser mais baixas do que as medianas obtidas por crianças com bom desempenho escolar nas pesquisas de Jacob (2001), Okano (2001) e Medeiros (2004). Das 23 comparações possíveis entre os resultados desta pesquisa e os daquelas autoras, 19 indicaram medianas menores na amostra de crianças com queixa escolar.

Os resultados das correlações entre as autopercepções e os escores dos demais instrumentos (Raven, TDE, CBCL). Indicam poucas correlações significativas, que se referem principalmente ao TDE: esse indicador correlacionou-se significativamente com os escores totais de autoconceito e autoeficácia, e também com a escala de autoconceito felicidade (Tabela 2). Esta última apresentou correlação, ainda, com o percentil no Raven.

As correlações entre os escores totais de autoconceito e autoeficácia e os subtestes do TDE foram baixas, porém significativas ou quase significativas.

Tabela 1. Medianas dos escores do autoconceito e autoeficácia, obtidas neste estudo e em estudos com crianças sem dificuldades escolares.

\begin{tabular}{|c|c|c|c|c|c|c|}
\hline \multirow{2}{*}{ Variável } & \multirow{2}{*}{ Mediana } & \multirow{2}{*}{ Mínimo } & \multirow{2}{*}{ Máximo } & \multicolumn{3}{|c|}{ Mediana } \\
\hline & & & & Medeiros $(2004)^{a}$ & Okano $(2001)^{\mathbf{b}}$ & Jacob $(2001)^{c}$ \\
\hline Comportamento & 13,0 & 4,0 & 16,0 & 14,0 & 14,0 & 15,0 \\
\hline Status intelectual e acadêmico & 12,0 & 2,0 & 17,0 & 15,0 & 14,0 & 16,0 \\
\hline Aparência & 11,0 & 1,0 & 13,0 & 11,0 & 10,5 & 12,0 \\
\hline Ansiedade & 9,0 & 1,0 & 13,0 & 11,5 & 9,0 & 12,0 \\
\hline Popularidade & 7,0 & 3,0 & 11,0 & 10,0 & 8,5 & 10,0 \\
\hline Felicidade & 8,0 & 3,0 & 10,0 & 9,0 & 9,0 & 9,5 \\
\hline Total & 58,0 & 21,0 & 74,0 & 69,0 & 65,0 & 74,0 \\
\hline Autoeficácia & 13,0 & 3,0 & 20,0 & 19,0 & - & 19,5 \\
\hline
\end{tabular}

${ }^{a}$ Crianças com bom desempenho escolar, $\mathrm{n}=26$, idade entre 8 e 12 anos; $^{\mathbf{b}}$ Crianças com bom desempenho escolar, $\mathrm{n}=20$, idade entre 7 e 10 anos; ${ }^{\mathrm{C}} \mathrm{Crianças}$ com bom desempenho escolar, $n=20$, idade entre 8 e 12 anos. 
Tabela 2. Correlações entre as medidas de autopercepção e os escores obtidos no Raven, TDE e CBCL.

\begin{tabular}{|c|c|c|c|c|c|c|}
\hline \multirow{2}{*}{ Variáveis } & \multirow{2}{*}{ Raven } & \multicolumn{4}{|c|}{ TDE } & \multirow{2}{*}{$\mathrm{CBCL}$} \\
\hline & & Escrita & Aritmética & Leitura & Total & \\
\hline \multicolumn{7}{|l|}{ Autoconceito } \\
\hline Comportamento & 0,22 & 0,16 & 0,16 & 0,19 & 0,18 & $-0,20$ \\
\hline Status intelectual e acadêmico & $-0,05$ & 0,09 & 0,10 & 0,10 & 0,13 & 0,08 \\
\hline Aparência & $-0,06$ & 0,09 & 0,11 & 0,14 & 0,15 & $-0,01$ \\
\hline Ansiedade & 0,24 & $0,29^{*}$ & $0,29^{*}$ & $0,25^{\dagger}$ & $0,28^{+}$ & $-0,02$ \\
\hline Popularidade & 0,24 & 0,22 & $0,29^{*}$ & 0,19 & 0,24 & $-0,02$ \\
\hline Felicidade & $0,38^{*}$ & $0,48^{* * *}$ & $0,37^{*}$ & $0,47^{* *}$ & $0,46^{* *}$ & $-0,10$ \\
\hline Total & $0,27^{\dagger}$ & $0,29^{*}$ & $0,29^{*}$ & $0,30^{*}$ & $0,31^{*}$ & $-0,06$ \\
\hline Autoeficácia & 0,18 & $0,29^{*}$ & $0,33^{*}$ & $0,28^{\dagger}$ & $0,32^{*}$ & $-0,07$ \\
\hline
\end{tabular}

${ }^{*} p \leq 0,05 ;{ }^{* *} p \leq 0,01 ;{ }^{\dagger} p<0,10$.

TDE: teste de desempenho escolar; CBCL: inventário de comportamentos da infância e adolescência.

Dentre as categorias de autoconceito, felicidade correlacionou-se consistentemente com desempenho nos subtestes do TDE, enquanto ansiedade correlacionou-se com desempenho em escrita e aritmética, apresentando uma correlação marginalmente significativa com leitura. Não se observaram correlações significativas envolvendo problemas de comportamento.

\section{Discussão}

Este estudo foi conduzido com o objetivo principal de caracterizar o autoconceito e o senso de autoeficácia de meninos com queixa escolar, encaminhados a uma clínica de psicologia vinculada ao Sistema Único de Saúde (SUS). Para alcançar esse objetivo, as autopercepções foram investigadas em relação a indicadores de funcionamento cognitivo, acadêmico e comportamental dos participantes.

\section{Funcionamento cognitivo, acadêmico e comportamental}

A maior parte das crianças apresentou potencial intelectual preservado, porém o TDE confirmou prejuízo no desempenho de $80 \%$ da amostra, enquanto o CBCL indicou 70\% dos participantes em situação de risco para problemas de comportamento. Tais resultados são consistentes com a literatura. Crianças com a queixa escolar, quando comparadas a seus pares, são avaliadas pelas mães e pelos professores como portadoras de mais comportamentos problemáticos (Jacob, 2001; Medeiros et al., 2000).

Em se tratando de meninos, como é o caso da amostra do presente estudo, há indícios de que eles exibem com maior frequência os comportamentos disruptivos associados aos transtornos da aprendizagem (APA, 1994). Essa circunstância poderia, inclusive, explicar o predomínio de meninos na população clínica com queixa escolar (Marturano et al., 2005).

\section{Autopercepções}

Os participantes, considerados como grupo, apresentaram autopercepções aparentemente mais desfavoráveis do que crianças da mesma idade com bom desempenho escolar, avaliadas em outros estudos com os mesmos instrumentos (Jacob, 2001; Medeiros, 2004; Okano, 2001). Dentro das limitações de uma análise baseada meramente na inspeção visual das medianas, os valores apresentados na Tabela 1 sugerem uma tendência consistente de menores resultados na amostra deste estudo: sete das oito comparações com resultados de Medeiros (2004) refletem essa tendência, assim como cinco das sete comparações com resultados de Okano (2001) e todas as comparações com os resultados de Jacob (2001).

Segundo a literatura que associa autoconceito, autoeficácia e desempenho acadêmico, seria esperado que, efetivamente, os participantes deste estudo apresentassem menor senso de autoeficácia acadêmica e pior autoconceito acadêmico (Jacob, 2001; Souza \& Brito, 2008; Valentine et al., 2004). Menos previsível, a partir dos estudos prévios, seriam as diferenças em outras categorias de autoconceito, já que se espera correspondência entre domínios de autopercepção e de performance (Marsh \& Craven, 2006). 
Por outro lado, as tendências identificadas na Tabela 1 são compatíveis com a visão da criança com queixa escolar, apresentada na introdução, como alguém vulnerável a autopercepções desfavoráveis, não só quanto ao desempenho, mas também quanto ao comportamento. Também convergem com os resultados de estudos empíricos que confirmam essa visão, mostrando, em crianças com queixa escolar, prejuízo do autoconceito nas categorias ansiedade, popularidade e felicidade (Medeiros, 2004; Okano, 2001; Stevanato et al., 2003).

\section{Correlações entre autopercepções e indicadores de desempenho e comportamento.}

A principal motivação para se realizar este estudo foi verificar se seriam reproduzidas as associações, encontradas na literatura, entre desempenho e autopercepções relacionadas à escola, bem como verificar se seriam encontradas correlações entre desempenho e autoconceito, nos domínios relacionados a comportamento, emoções e relações interpessoais, conforme sugerido por estudos prévios sobre crianças com queixa escolar.

As correlações modestas, porém significativas, entre o autoconceito global e todas as medidas derivadas do TDE corroboram os resultados encontrados por outros autores, que constataram relação direta entre autoconceito global e desempenho escolar em alunos do ensino fundamental (Cia \& Barham, 2009; Silva \& Alencar, 1984). No entanto, em relação ao autoconceito acadêmico, as correlações encontradas não foram significativas, o que diverge dos resultados obtidos em pesquisas prévias (Carneiro et al., 2003; Jacob, 2001; Marsh \& Craven, 2006; Medeiros et al., 2000; Moraes \& Cunha, 2007; Okano et al., 2004; Stevanato et al., 2003).

Como explicar essa discrepância? Poderiam ser evocadas diferenças na composição das amostras. A amostra deste estudo pode ser considerada mais homogênea que a das pesquisas precedentes, não apenas quanto ao gênero, mas também quanto ao desempenho rebaixado no TDE. Em amostras homogêneas, as diferenças individuais se atenuam, o que pode ter contribuído para a ausência de correlação no teste estatístico. Porém, essa explicação não parece inteiramente satisfatória, já que a medida de autoeficácia, com foco na eficácia acadêmica, mostrou correlação com escores de desempenho, confirmando a associação detectada em estudos com amostra heterogênea quanto a gênero, idade e série (Souza \& Brito, 2008) ou com grupos contrastados quanto ao desempenho (Jacob, 2001; Medeiros et al., 2000). Vale notar que, sendo o senso de autoeficácia mais dependente do contexto específico em que a pessoa faz o autojulgamento (Bong \& Skaalvik, 2003), talvez o contexto da avaliação em que se deu a pesquisa tenha influenciado a autoeficácia acadêmica das crianças de acordo com grau de sucesso percebido naquela tarefa, ao passo que o autoconceito acadêmico, mais estável, não foi diferencialmente afetado pela situação.

Assim como não foram encontradas as associações previstas entre desempenho e autoconceito acadêmico, também não se confirmou a associação entre problemas de comportamento e autoconceito no domínio comportamental, o que contradiz os achados da literatura (Jacob, 2001; Medeiros, 2004). Por outro lado, encontraram-se algumas correlações entre desempenho e autoconceito em domínios relacionados às emoções, conforme sugerido por estudos prévios sobre crianças com queixa escolar (Medeiros, 2004; Okano et al., 2004; Stevanato et al., 2003).

As correlações encontradas entre desempenho e autoconceito em domínios relacionados a felicidade e ansiedade remetem de novo à noção de vulnerabilidade associada às autopercepções de crianças com queixa escolar. Uma condição de vida recente, comum a todos os participantes do estudo, foi a procura de tratamento psicológico na rede SUS, motivada pela queixa escolar. Essa condição constitui evento adverso na história escolar da criança e, como tal, pode ter um impacto emocional importante em suas autopercepções, além da imagem que ela faz de si mesma como estudante.

A circunstância de os participantes terem sido avaliados no contexto de uma clínica procurada pela família, em razão de sua dificuldade escolar, pode ter contribuído para potencializar a interferência de estados emocionais nas avaliações do autoconceito. Nas condições em que os dados foram obtidos, as crianças responderam à Escala de Autoconceito logo depois da aplicação do TDE. Desse modo, pode-se supor um efeito da atividade acadêmica recém-concluída sobre as respostas de autopercepção.

Nesse sentido, uma leitura das correlações entre o desempenho escolar e o autoconceito felicidade pode 
ser formulada nos seguintes termos: quanto mais elevado o desempenho alcançado pela criança naquele momento, mais feliz ela se sentiu, refletindo isso em suas respostas na escala de autoconceito. Uma interpretação semelhante pode ser dada à associação entre o desempenho acadêmico e o autoconceito ansiedade, observada principalmente com relação às áreas da escrita e da aritmética do TDE: quanto mais baixo o desempenho alcançado pela criança naquele momento, mais angustiada ela se sentiu.

Pode-se supor que a correlação entre o autoconceito felicidade/ansiedade e o desempenho esteja refletindo o impacto cumulativo da história prévia de fracasso escolar no autoconceito, sobretudo naqueles domínios pessoais de bem-estar subjetivo ou sofrimento psíquico. Corroborando esses resultados, o autoconceito no domínio ansiedade da Escala Piers-Harris também discriminou crianças com queixa escolar, em estudos conduzidos em contexto de atendimento, seja na escola (Okano et al., 2004) ou na clínica (Medeiros, 2004; Stevanato et al., 2003).

A autoavaliação de maior ansiedade associada ao desempenho mais baixo, junto com a correlação significativa entre a autoeficácia e os indicadores de desempenho do TDE, faz refletir a respeito do círculo vicioso que isso representa, já que o senso de autoeficácia acadêmica prediz aprendizado e desempenho escolar (Carrol et al., 2009; Fast et al., 2010). Se já nas primeiras séries do ensino fundamental a criança acumular uma história de fracasso na aprendizagem, poderá desenvolver um baixo senso de autoeficácia acadêmica.

Não acreditando na própria capacidade, a criança tende a desistir diante das tarefas escolares, o que provavelmente resulta em desempenho ruim, reforçando ainda mais seu sentimento de incapacidade. $\mathrm{E}$ assim recomeça o ciclo - quanto mais a criança se sente incapaz, mais estará suscetível ao insucesso, cujos desdobramentos na escola e em casa geram ansiedade e sentimentos de infelicidade. Ou seja, uma vez que a criança desenvolve um baixo senso de autoeficácia, torna-se difícil romper esse círculo vicioso sem ajuda.

\section{Limites do trabalho}

Este foi um estudo correlacional, cujos resultados, 450 por isso mesmo, devem ser vistos como indicativos de associação e não de relações de causa e efeito. Essa ressalva é tanto mais pertinente quando se sabe que a relação entre autopercepções e desempenho é recíproca (Marsh \& Craven, 2006). As correlações encontradas foram em geral baixas, o que sugere associação fraca, analogamente a outros estudos (Cunha et al., 2006, 2007; Jacob, 2001; Moraes \& Cunha, 2007). Para melhor esclarecer a questão das autopercepções associadas à queixa escolar, sugere-se um delineamento de três grupos - um com bom desempenho, outro com desempenho pobre e um terceiro com desempenho pobre associado à condição de atendimento psicopedagógico fora da sala de aula.

\section{Considerações Finais}

Esta investigação replicou parcialmente, em amostra de meninos com queixa escolar, resultados obtidos anteriormente em amostras mistas quanto ao gênero, no que diz respeito à associação entre desempenho escolar, autoconceito e autoeficácia. Permanece aberta à investigação a questão da maior vulnerabilidade das crianças com queixa escolar para autopercepções desfavoráveis.

A pesquisa agregou à evidência disponível informações que confirmam, dentro de limites, a suscetibilidade dessas crianças a um contexto de avaliação de habilidades acadêmicas. Com efeito, a associação entre desempenho e autopercepções de sentimentos e emoções parece ser específica, encontrada apenas em grupos com queixa escolar. Para as autoras, essa é a principal contribuição do estudo.

\section{Referências}

Achenbach, T. M. (1991). Manual for the child behavior check-list/4-18 and 1991 profile. Burlington: University of Vermont.

American Psychiatric Association. (1994). Diagnostic and statistical manual of mental disorder ( $4^{\text {th }}$ ed.). Washington, DC: Author.

Angelini, A., Alves, I., Custódio, E., \& Duarte, W. (1999). Manual das matrizes progressivas coloridas. São Paulo: Casa do Psicólogo.

Bandura, A. (2006). Toward a psychology of human agency. Perspectives on Psychological Science, 1 (2), 164-180.

Bong, M., \& Skaalvik, E. M. (2003). Academic self-concept and self-efficacy: how different are they really? Educational Psychology Review, 15 (1), 1-40. 
Bordin, I. A. S., Mari, J. J. E., \& Caeiro, M. F. (1995). Validação da versão brasileira do "Child Behavior Checklist" (CBCL) Inventário de comportamento da infância e adolescência: dados preliminares. Revista ABP-APAL, 17 (1), 55-56.

Candieux, A. (1996). Psychometric properties of a pictorial self-concept scale among young learning disabled pupils. Psychology in the Schools, 33 (3), 221-229.

Carneiro, G. R. S., Martinelli, S. C., \& Sisto, F. F. (2003). Autoconceito e dificuldades de aprendizagem na escrita. Psicologia: Reflexão e Crítica, 16 (3), 427-434.

Carrol, A., Houghton, S., Wood, R., Unsworth, K., Hattie, J., Gordon, L., et al. (2009). Self-efficacy and academic achievement in Australian high school students: the mediating effects of academic aspirations and delinquency. Journal of Adolescence, 32 (4), 797-817.

Cia, F., \& Barham, E. (2009). Repertório de habilidades sociais, problemas de comportamento, autoconceito e desempenho acadêmico de crianças no início da escolarização. Estudos de Psicologia (Campinas), 26 (1), 45-55. doi: 10.1590/S0103-166X2009000100005.

Cunha, C. A. , Sisto, F. F., \& Machado, F. (2006). Dificuldade de aprendizagem na escrita e o autoconceito num grupo de crianças. Avaliação Psicológica, 5 (2), 153-157.

Cunha, C. A. , Sisto, F. F., \& Machado, F. (2007). Autoconceito e reconhecimento de palavras em crianças do ensino fundamental. Avaliação Psicológica, 6 (2), 147-156.

Donohue, D., Wise, J. C., Romski, M., Henrich, C. C., \& Sevcik, R. A. (2010). Self-concept development and measurement in children with mild intellectual disabilities. Developmental Neurorehabilitation, 13 (5), 322-334.

Fast, L. A., Lewis, J. L., Bryant, M., Bocian, K. A., Cardullo, R. A., Rettig, M., et al. (2010). Does math self-efficacy mediate the effect of the perceived classroom environment on standardized math test performance? Journal of Educational Psychology, 102 (3), 729-740.

Fontaine, A. M. (1995). Expectativas e realização escolar: o efeito "pigmaleão" questionado. Revista Portuguesa de Pedagogia, 29 (1), 119-132.

Gest, S. D., Rulison, K. L., Davidson, A. J., \& Welsh, J. A. (2008), A reputation for success (or failure): the association of peer academic reputations with academic self-concept, effort, and performance across the upper elementary grades. Developmental Psychology, 44 (3), 625-636.

Jacob, A. V. (2001). O desempenho escolar e suas relações com autoconceito e auto-eficácia. Tese de doutorado não- publicada, Programa de Pós-Graduação em Saúde Mental, Universidade de São Paulo, Ribeirão Preto.

Loureiro, S. R., \& Medeiros, P. C. (2004). O senso de auto-eficácia de crianças com dificuldades de aprendizagem. In E. M. Marturano, M. B. M. Linhares \& S. R. Loureiro (Orgs.), Vulnerabilidade e proteção: indicadores na trajetória de desenvolvimento do escolar (pp.179-195). São Paulo: FAPESP.

Martin, E., \& Marchesi, A. (1995). Da terminologia dos distúrbios às necessidades educacionais especiais. In C. Coll, J. Palácios \& A. Marchesi (Orgs.), Desenvolvimento psicológico e educação: necessidades educativas especiais e apren- dizagem escolar (Vol. 3, pp.17-35). Porto Alegre: Artes Médicas.

Marturano, E. M., Toller, G. P., \& Elias, L. C. S. (2005). Gênero, adversidade e problemas sócio-emocionais associados à queixa escolar. Estudos de Psicologia (Campinas), 22 (4), 371-380. doi: 10.1590/S0103-166×2005000400005.

Marsh, H. W., \& Craven, R. G. (2006). Reciprocal effects of self-concept and performance from a multidimensional perspective: beyond seductive pleasure and unidimensional perspectives. Perspectives on Psychological Science, 1 (2), 133-163.

Medeiros, P. C. (2004). Crianças com dificuldade de aprendizagem: vulnerabilidade e proteção associados ao comportamento, às autopercepções e ao suporte psicopedagógico. Tese de doutorado não-publicada, Programa de Pós-Graduação em Psicologia, Universidade de São Paulo, Ribeirão Preto.

Medeiros, P.C., Loureiro, S. R., Linhares, M. B. M., \& Marturano, E. M. (2000). A auto-eficácia e os aspectos comportamentais de crianças com dificuldade de aprendizagem. Psicologia: Reflexão e Crítica, 13 (3), 328-333.

Moraes Jr, R., \& Cunha, C. A. (2007). Reconhecimento de palavras e autoconceito num grupo de crianças. Psic: Revista da Vetor Editora, 8 (2), 215-226.

Okano, C. B. (2001). O autoconceito de crianças atendidas em um programa de suporte psicopedagógico na escola. Dissertação de mestrado não-publicada, Universidade de São Paulo, Ribeirão Preto.

Okano, C. B., Loureiro, S. R., Linhares, M. B. M., \& Marturano, E. M. (2004). Crianças com dificuldades escolares atendidas em programa de suporte psicopedagógico na escola: avaliação do autoconceito. Psicologia: Reflexão e Crítica, 17 (1), 121-128.

Silva, I. V., \& Alencar, E. M. L. S. (1984). Autoconceito, rendimento academico e escolha do lugar de sentar entre alunos de nivel socio-econômico medio e baixo. Arquivos Brasileiros de Psicologia, 36 (1), 89-96.

Silva, S. S., \& Fleith, D. S. (2005). Desempenho escolar e autocenceito de alunos atendidos em serviços psicopedagógicos. Psicologia Escolar e Educacional, 9 (2), 235-245.

Souza, L. F. N. I., \& Brito, M. R. F. (2008).Crenças de autoeficácia, autoconceito e desempenho em matemática. Estudos de Psicologia (Campinas), 25 (2), 193-201. doi: 10.1590/S0103-166X2008000200004.

Stevanato, I. S., Loureiro, S. R., Linhares, M. B. M., \& Marturano, E. M. (2003). Autoconceito de crianças com dificuldades de aprendizagem e problemas de comportamento. Psicologia em Estudo, 8 (1), 67-76.

Stein, L. M. (1984). TDE: teste do desempenho escolar: manual para aplicação e interpretação. São Paulo: Casa do Psicólogo.

Valentine, J. C., DuBois, D. L., \& Cooper, H. (2004). The relation between self-beliefs and academic achievement: a systematic review. Educational Psychologist, 39 (1), 111-133.

Recebido em: 8/5/2009

Versão final reapresentada em: 5/5/2011

Aprovado em: 11/5/2011 
